\title{
Recent advanced in Surface Guided Radiation Therapy
}

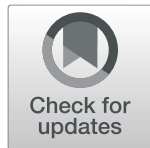

P. Freislederer ${ }^{{ }^{*}} \mathbb{D}$, M. Kügele ${ }^{2,3}$, M. Öllers ${ }^{4}$, A. Swinnen ${ }^{4}$, T.-O. Sauer ${ }^{5}$, C. Bert ${ }^{5}$, D. Giantsoudi ${ }^{6}$, S. Corradini ${ }^{1}$ and V. Batista ${ }^{7,8,9}$

\begin{abstract}
The growing acceptance and recognition of Surface Guided Radiation Therapy (SGRT) as a promising imaging technique has supported its recent spread in a large number of radiation oncology facilities. Although this technology is not new, many aspects of it have only recently been exploited. This review focuses on the latest SGRT developments, both in the field of general clinical applications and special techniques.

SGRT has a wide range of applications, including patient positioning with real-time feedback, patient monitoring throughout the treatment fraction, and motion management (as beam-gating in free-breathing or deep-inspiration breath-hold). Special radiotherapy modalities such as accelerated partial breast irradiation, particle radiotherapy, and pediatrics are the most recent SGRT developments.

The fact that SGRT is nowadays used at various body sites has resulted in the need to adapt SGRT workflows to each body site. Current SGRT applications range from traditional breast irradiation, to thoracic, abdominal, or pelvic tumor sites, and include intracranial localizations.

Following the latest SGRT applications and their specifications/requirements, a stricter quality assurance program needs to be ensured. Recent publications highlight the need to adapt quality assurance to the radiotherapy equipment type, SGRT technology, anatomic treatment sites, and clinical workflows, which results in a complex and extensive set of tests.

Moreover, this review gives an outlook on the leading research trends. In particular, the potential to use deformable surfaces as motion surrogates, to use SGRT to detect anatomical variations along the treatment course, and to help in the establishment of personalized patient treatment (optimized margins and motion management strategies) are increasingly important research topics. SGRT is also emerging in the field of patient safety and integrates measures to reduce common radiotherapeutic risk events (e.g. facial and treatment accessories recognition).

This review covers the latest clinical practices of SGRT and provides an outlook on potential applications of this imaging technique. It is intended to provide guidance for new users during the implementation, while triggering experienced users to further explore SGRT applications.
\end{abstract}

Keywords: Surface guided radiation therapy, SGRT, Patient positioning, Motion management, Deep-inspiration breath-hold, Intra-fractional motion mitigation, Patient safety

\footnotetext{
* Correspondence: philipp.freislederer@med.uni-muenchen.de

'Department of Radiation Oncology, University Hospital, LMU Munich,

Munich, Germany

Full list of author information is available at the end of the article
}

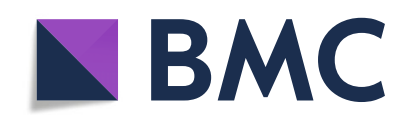

(c) The Author(s). 2020 Open Access This article is licensed under a Creative Commons Attribution 4.0 International License, which permits use, sharing, adaptation, distribution and reproduction in any medium or format, as long as you give appropriate credit to the original author(s) and the source, provide a link to the Creative Commons licence, and indicate if changes were made. The images or other third party material in this article are included in the article's Creative Commons licence, unless indicated otherwise in a credit line to the material. If material is not included in the article's Creative Commons licence and your intended use is not permitted by statutory regulation or exceeds the permitted use, you will need to obtain permission directly from the copyright holder. To view a copy of this licence, visit http://creativecommons.org/licenses/by/4.0/ The Creative Commons Public Domain Dedication waiver (http://creativecommons.org/publicdomain/zero/1.0/) applies to the data made available in this article, unless otherwise stated in a credit line to the data. 


\section{Introduction}

In recent years, the clinical use of Surface Guided Radiation Therapy (SGRT) using optical surface scanning for patient positioning, intra-fraction motion monitoring and respiratory gating techniques has increased. In general, SGRT systems use a combination of a projector and one or several camera units to register a real-time $3 \mathrm{D}$ surface of the patients. A reference surface relative to the treatment isocenter position is used to calculate the necessary correction of the patient position in translational and rotational directions. There are four main optical surface scanning technologies used in radiotherapy, laser scanners [1], time-of-flight systems [2, 3], stereovision systems [4] and structured light systems [5, 6]. Optical surface scanners, with their high spatial and temporal resolution, have proven to be an important addition to the radiation therapy process regarding patient positioning and monitoring $[1,7,8]$.

SGRT can be seen as a "four-eyes principle" tool that allows continuous monitoring of patient positioning, thus improving patient safety $[9,10]$ and comfort $[11,12]$, and at the same time standardizing workflows (higher precision and reproducibility) [13]. Additionally, it has the potential to improve the clinical outcomes through accurate target irradiation [14], while sparing normal tissue [15].

In terms of patient positioning, SGRT is an effective tool for the reduction of the overall treatment time, and minimizing of imaging dose, as: i) it provides in-room online information of the complete surface and position of the patient, ii) for superficial tumors (where surface deviations can act as a surrogate for tumor motion) SGRT allows for a more accurate positioning compared to 3-point-lasers and might allow to reduce the number of daily imaging in some cases [16]; iii) for deeper located tumors (with no direct correlation between surface deviations and tumor movement) daily imaging remains mandatory, but SGRT can reduce the time required for image registration and prevent the need for multiple imaging [17]. Although imaging dose could be seen as negligible compared to the whole body scatter dose from a photon treatment, SGRT can be identified as one imageguided step that can be accomplished without ionizing radiation as proposed in AAPM TG 75 for imaging dose reduction [18].

As SGRT systems provide real-time motion monitoring of the patient surface throughout the whole treatment fraction, an additional level of safety is added to radiotherapy workflows. The beam can be held if parts of the patient's surface deviate from the reference position based on the planning CT set-up or if the calculated isocentric deviations exceed a certain threshold.

One of the most promising applications of SGRT is gated radiotherapy delivery of tumor locations close to the skin surface (e.g. breast cancer) by means of Deep-
Inspiration-Breath-hold (DIBH) and voluntary-DIBH (vDIBH). Another application developed in recent years is the use of SGRT in whole-brain radiation therapy (WBRT) or stereotactic radiosurgery (SRS). Here, an SGRT system has the ability to monitor the surface of the patient within an open-face immobilization mask in planar and non-coplanar treatments [19]. SGRT has also been applied in special techniques such as accelerated partial breast irradiation (APBI), stereotactic body radiation therapy (SBRT), and with limited use in pediatrics [20].

The present review aims to provide a summary of recent clinical advances in SGRT and the latest research findings applied to modern SGRT-based treatments, with an outlook on future application fields.

\section{Clinical applications}

SGRT has become a common tool in radiotherapy due to its ability to provide real-time, 6-dimensional patient positioning and monitoring, as exemplarily illustrated in Fig. 1. Daily imaging of the patient without concern for radiation dose and the potential for reduced setup times are the main advantages of the technology. In order to identify the clinical benefits of surface imaging for patient setup, several investigations have been conducted which benchmark the patient setup accuracy using verification imaging. There is a broad agreement on the supremacy of SGRT over 3-point laser-based patient setup, when the surface serves as a surrogate for the target positioning, i.e. breast and other skin-near targets $[13,16$, 21-30]. For deeper situated targets, surface imaging should be used with caution [13, 22, 31, 32].

\section{Breast positioning}

Regarding setup of breast cancer patients, studies comparing laser alignment with surface imaging report a reduction of positioning errors for skin and clip alignment [23-26] by around $40 \%$ on average, with absolute errors (1SD) for all studies being smaller than $4 \mathrm{~mm}$, verified with $\mathrm{kV}$ orthogonal imaging [29, 30, 33, 34], portal imaging $[16,27,30]$, or cone-beam computed tomography (CBCT) [13]. In addition to accurate isocenter positioning, the surface imaging provides guidance for correcting patient posture, i.e. chin and arm position. In this context, it has been shown that surface-guided correction of the arm posture also improved the breast position [13, $16,27,30]$. Nevertheless, some factors can affect the accuracy in surface-guided patient setup, such as patient motion [35], surface shadowing [16], selection of the region-of interest (ROI) [14], absence of anatomical gradients in the patient (e.g. very flat surfaces) and anatomical changes throughout the treatment $[21,28,36]$. The SGRT workflow within an NAL (No Action Level) imaging protocol has shown the potential to reduce the frequency of IGRT, and thus spare additional imaging dose 

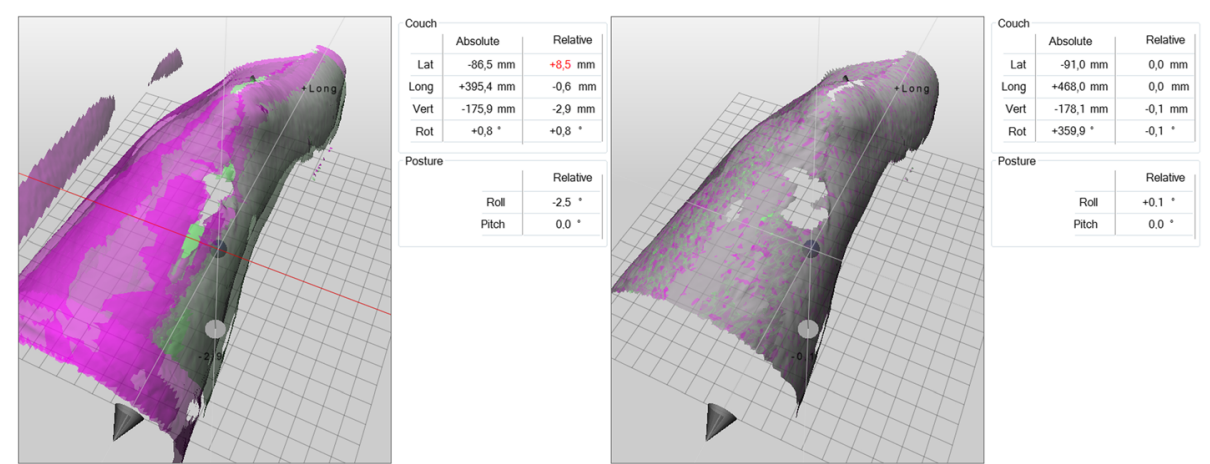

Fig. 1 Example of patient positioning using SGRT. Left: The live surface data from a whole left leg (purple) deviates from the reference surface (green). Right: After correction, the surface data matches the reference image. Image courtesy of LMU University Hospital Munich, Germany

$[16,30,31]$. Advances in surface imaging for positioning of breast cancer patients have recently led to a tattoofree workflow and reduced time for patient setup compared to laser alignment [26].

\section{Positioning of internal targets (abdomen/pelvis/Head\& Neck)}

Several studies have shown a poor correlation between movement of the patient surface and movement of internal targets [13, 22, 37, 38]. Large shifts up to about 3 $\mathrm{cm}$ were observed for targets in the abdomen, and up to about $2 \mathrm{~cm}$ for pelvis and lower extremities [13, 22, 31]. However, surface imaging achieved at least the same precision as laser alignment and was considered a valuable tool for initial patient setup and as a complement to conventional imaging modalities [13, 22, 38]. The lack of correlation between the surface and internal structure movement is not an argument against the use of SGRT [32], since SGRT does not provide contradictory but complementary information for image guidance. The choice of the ROI for the reference image influences the correlation between SGRT and image-guided positioning. In pelvic SGRT, improved agreement with CBCT was found by excluding deformable anatomies such as the stomach from the reference image ([39]). In contrast, only minor displacements were observed in the head and neck region [22, 31]. Haraldsson et al. reported significantly reduced imaging times for patient positioning using surface imaging in head and neck patients by 5 min per fraction [31].

\section{Intracranial tumors: WBRT \& SRS}

Traditionally, WBRT has been the recommended treatment option for patients with more than three intracranial brain metastases (BM), but WBRT can cause longterm adverse events (e.g. neurocognitive decline) and a reduced quality of life [40]. In contrast, for a limited number of metastases [1-4], stereotactic radiosurgery is the option of first choice in most cases. Recent technological advances have made linac-based frameless SRS a more patient friendly treatment option, allowing for accurate patient positioning and shorter treatment times [41-45]. Since SRS is increasingly used in patients with multiple BM, it is important that SRS is performed with the highest quality achievable to avoid complications such as radionecrosis. Therefore, it is essential to treat the $\mathrm{BM}$ with smaller treatment margins, which requires a 6DOF correction under the guidance of onboard CBCT and a thermoplastic mask [46]. This improved patient setup makes it possible to treat multiple lesions with a single isocenter, which in combination with flattening filter free (FFF) beams can further decrease the beam-on time $[47,48]$.

The advantages of including non-coplanar couch angles in the treatment planning can result in better sparing of normal brain tissue. This approach has already been extensively published $[47,49]$. However, with modern Linacs it is not possible to verify the patient's position using CBCT at non-coplanar couch angles (Fig. 2). Additional equipment such as the ExacTrac ${ }^{\circ} \mathrm{X}$-ray system (Brainlab AG, Munich, Germany) is able to provide accurate intrafraction setup information of the bony anatomy of the patient - regardless of the couch angle, but the imaging procedure adds some additional dose and extends the overall treatment time [50, 51]. Tarnavski et al. showed that although patients were immobilized with thermoplastic masks, positioning corrections exceeding $1 \mathrm{~mm}$ appeared in $42 \%$ of the beams and exceeding 1 degree in $9 \%$ of the beams [52].

A clear advantage of SGRT systems is the lack of radiation dose and the procedure only minimally increases the overall treatment time [8]. Optimal use of SGRT systems in patients with brain tumors requires the use of open-face masks or even no masks. Dekker et al. described the results of 30 palliative patients treated with WBRT without any immobilization mask [53]. With a success rate of $93 \%$, the authors concluded that radiation therapy without a thermoplastic mask was clinically 

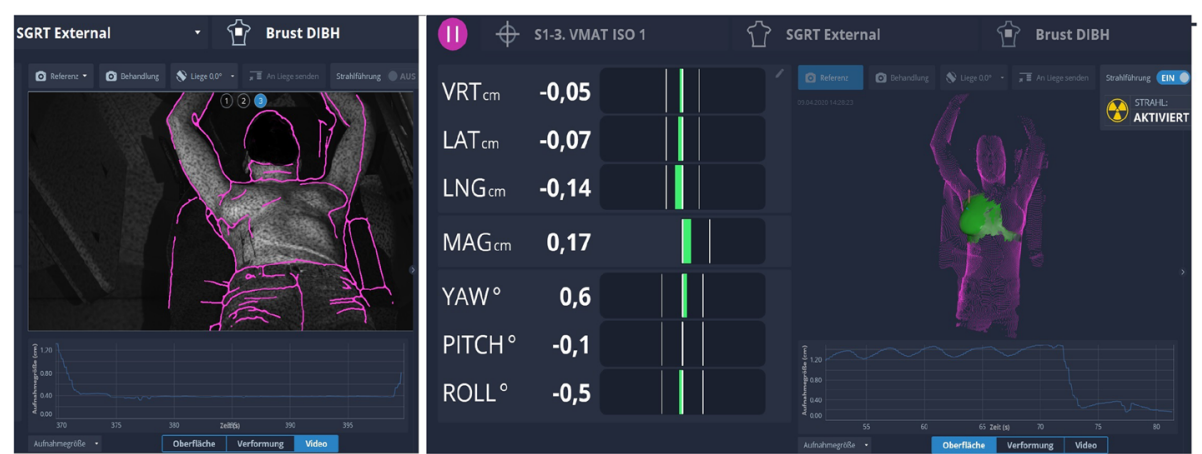

Fig. $\mathbf{2}$ a ORFIT open face mask together with a T-shaped vacuum bag, b Catalyst HDTM in kV-MV setup using the ExaFix-3 baseplate and $\mathbf{c}$ in setup at couch $0^{\circ}(3$ cameras are indicated with arrows). The cropped surface image (d) is extracted from the patient's open face mask (e). Image courtesy of MAASTRO Clinic, Maastricht, The Netherlands

feasible. Under guidance of a single camera system (CRAD, Uppsala, Sweden), the isocenter motion was within $1.1 \mathrm{~mm}$ on average. Obviously, the system is interconnected with the Linac and can perform an automated beam shut off if the patient moves out of a predefined threshold.

For treatments with non-coplanar couch angles, accurate knowledge about the coincidence between radiation and couch rotation isocenter becomes important, as it is not possible to correct for couch walkout during treatment delivery. Regular Winston-Lutz checks provide accurate information about the radiation isocenter for different gantry, collimator and couch angles and can also indirectly provide information about the accuracy of the system $[19,54,55]$.

\section{Breath-hold}

Since respiratory-induced organ motion is considered the largest intrafractional organ motion, the uncertainty during radiation therapy of tumors or lesions which are affected by respiration has to be taken into account. DIBH (maximum inspiration) or a shallow BH (moderate inspiration) are effective methods of respiratory motion mitigation [56]. Both concepts reduce tumor motion to a minimum and allow for a reduction in dose to the heart with similar planning target volume (PTV) coverage [57]. For lung or liver treatments, the breathhold level can be monitored using SGRT systems, although the system can only monitor the patient surface as a surrogate for tumor motion at most. On the other hand, respiratory motion can also be used to increase OAR sparing for specific tumor sites, in which DIBH increases the distance between the PTV and the OARs [58]. For example, in breast cancer the DIBH technique leads to a reduction in cardiac dose, especially to radiosensitive structures like the left anterior descending artery (LAD) where any additional dose increases the risk of coronary artery disease and risk of ischemic heart disease [59-63]. Laaksomaa et al. reported the possibility of portal imaging reduction for whole breast DIBH with residual errors of $\leq 3 \mathrm{~mm}$ [30]. Furthermore, DIBH reduces the interplay effect and improves plan robustness [56] and reduces the dose to LAD and heart significantly in proton therapy for breast [64] and Hodgkin's lymphoma [65]. Left breast cancer irradiation using DIBH using optical surface scanners is nowadays widely implemented at multiple institutions [66] and the concept has also been adapted to right-sided breast irradiation for a reduction of lung and liver dose [67].

SGRT systems offer the possibility of evaluating intraDIBH stability, which has been reported with $\leq 0.7 \mathrm{~mm}$, together with an intra-fractional reproducibility of $\leq 2.2$ $\mathrm{mm}$ [68] and with $0.3 \mathrm{~mm}$ as the median standard deviation of the BH level during DIBH [69]. Kügele et al. reported that the intrafractional reproducibility for tangential and locoregional treatment was as low as 1 $\mathrm{mm}$ (median over 40 patients) in all three translational directions, but during a single treatment session the maximum deviation was up to $5 \mathrm{~mm}$, which resulted in large effects on the target coverage and OAR doses [70]. The accuracy of SGRT systems have been reported within $5 \mathrm{~mm}$ for DIBH positioning and monitoring [71], and are similar to those reported in studies using spirometry-based positioning [68].

A reduction in treatment time using SGRT for DIBH has not been consensually reported, but an improved safety aspect as patient positioning, intra-DIBH stability, and intrafractional DIBH reproducibility can be measured directly and accounted for using an adaption of setup margins and beam control. Figures 3 and 4 show examples of DIBH patient positioning and monitoring.

\section{Special techniques}

Accelerated partial breast irradiation (APBI).

In APBI the treatment target is limited to the postsurgical cavity of patients with early-stage breast cancer 


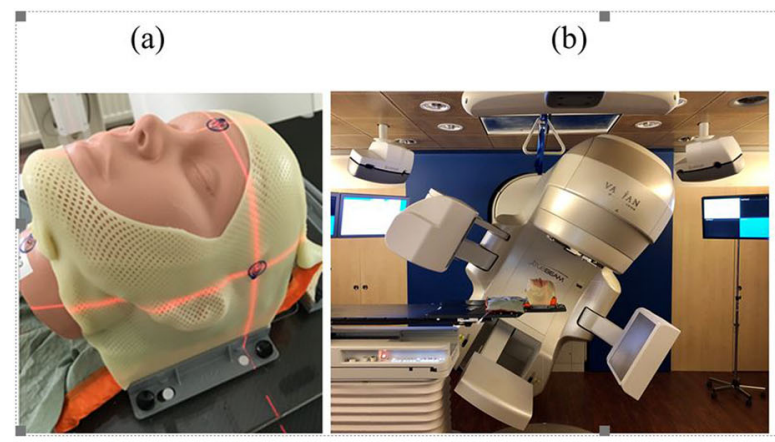

(d) (c)

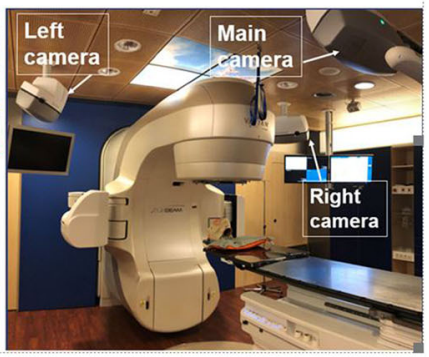

(e)

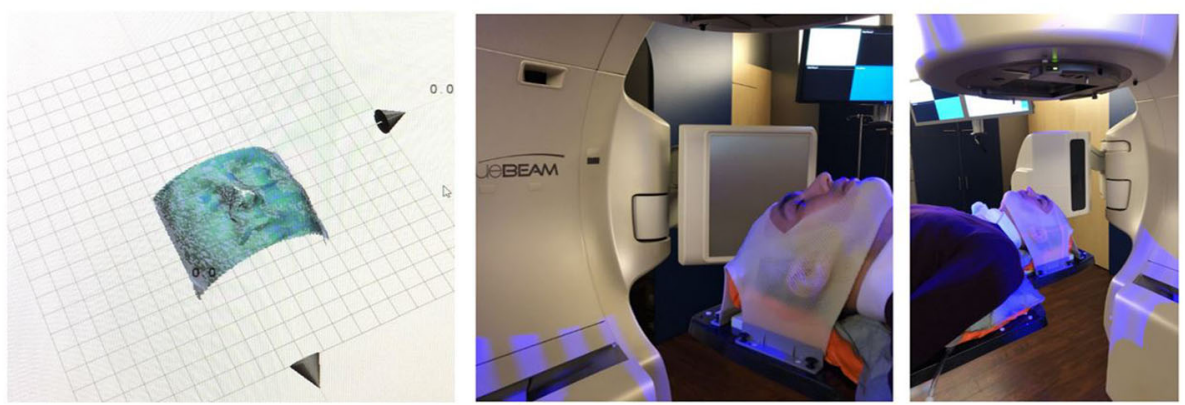

Fig. 3 Positioning of a DIBH patient. Left: Positioning of the patient using the reference surface (purple) and the live surface data. Right: Monitoring of the DIBH during treatment on a highlighted (green) ROI. The breathing curve is depicted on the bottom. Image courtesy of Heidelberg University Hospital, Germany

where surgical clips can be implanted for target localization via $x$-ray imaging. SGRT has been shown to improve setup for this soft tissue target [72] and a strong correlation of the external patient surface with the internal resection site has been suggested [24]. In a recent study, the target registration error (TRE) for a SGRT system agreed with the $\mathrm{kV}$ clip-based TRE within less than $3 \mathrm{~mm}$. Furthermore, the combination of SGRT and 2D X-ray matching to surgical clips allowed for accurate alignment and setup verification without the need for skin-based tattoos [26]. In the absence of surgical clips in the lumpectomy area, SGRT still appears to be a potentially valuable image guidance approach for patients treated with APBI when they experience $<10 \%$ changes in surgical cavity volume between computed tomography $(\mathrm{CT})$ simulation and treatment [21].

\section{Motion management and SBRT}

Almost one third of all clinical SGRT users have implemented respiratory gating for SBRT treatments or conventional lung or abdominal $\mathrm{RT}$, for patient positioning and/or motion management [32]. SGRT can improve pre-imaging treatment setup, decrease the necessity of orthogonal $\mathrm{kV}$ imaging prior to $\mathrm{CBCT}$ [73] and is promising in detecting deviations between pre- and posttreatment setup in SBRT [74]. Four-dimensional computed tomography (4DCT) acquisition and treatment delivery under surface guidance showed excellent temporal accuracy and consistency to the Varian RPM system in motion-tracking studies [75-77] and has the potential to improve respiratory motion management [78]. Kauweloa et al. observed an increased uncertainty in mean-position tracking of surface-guided 4DCTacquisition with decreasing amplitude of the breathing pattern, suggesting that the SGRT system might be more appropriate for phase- rather than amplitude-sorting of 4DCT [76]. The potential of SGRT for respiratory motion monitoring and motion management is demonstrated by the strong correlation of SGRT monitoring with internal tumor position monitoring by $\mathrm{x}$-ray imaging $[79,80]$. Respiratory motion signal and estimated volume variations are well correlated with spirometer measurements [81-83]. However, when implementing a SGRT-based tumor-tracking or gating-system, careful characterization of the beam-on and beam-off delays is advisable, as these might be non-negligible and vary between the SGRT and beam delivery systems [84-86].

\section{Pediatric}

SGRT-based intra-fraction monitoring of pediatric treatments is not widely used [32] and literature is limited. In a case report [87] using SGRT in combination with a linac using the FFF mode, the palliative radiation treatment of an 18-month old boy with relapsed Wilms 

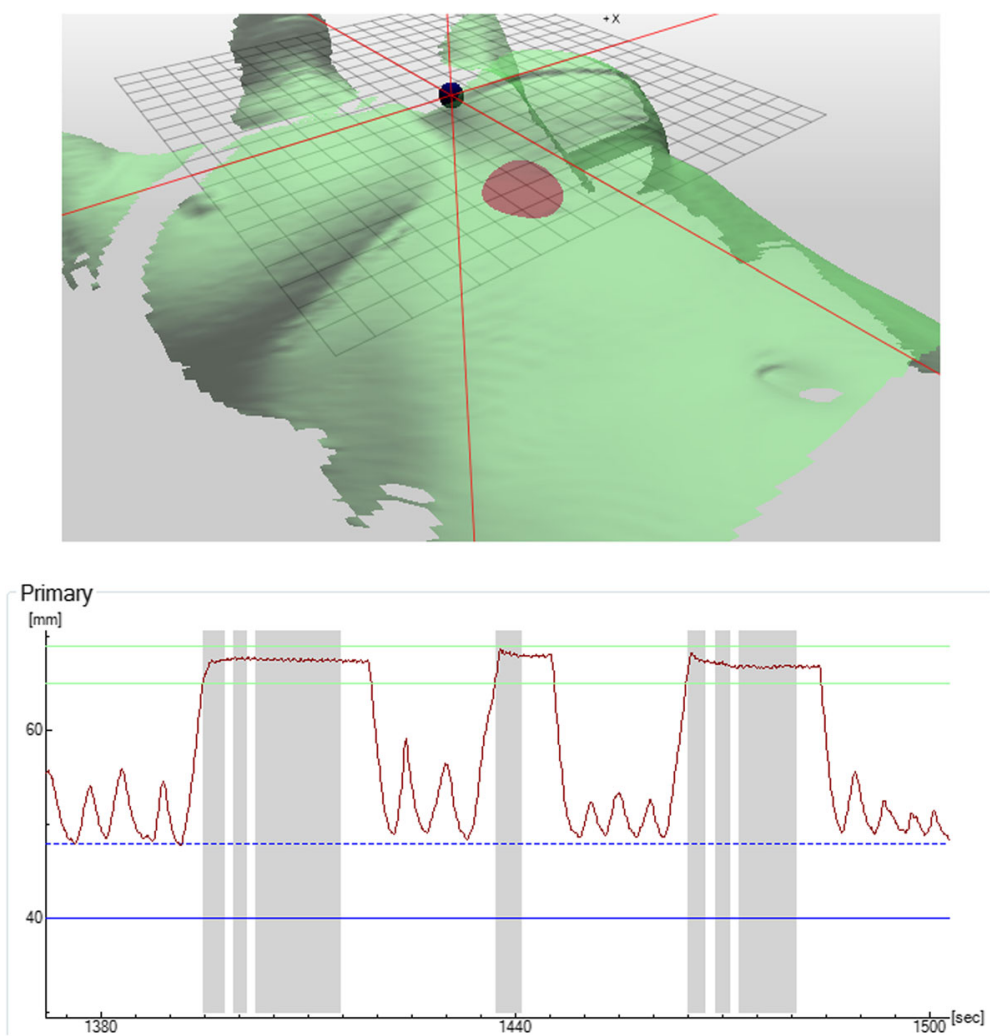

Fig. $4 \mathrm{DIBH}$ monitoring. Top: A breathing spot (red) on the patient surface (green) is monitored. Bottom: The breathing curve with three breathholds, covering 7 individual beams (indicated using the grey bars). Image courtesy of LMU University Hospital Munich, Germany

tumor was reported. He had a large anterior mediastinal mass which critically obstructed his airway. SGRT treatment could be delivered in sufficiently short time without anesthesia. SGRT systems have been added as a safety feature in pediatric treatments to assist in patient setup and provide additional error detection [88].

\section{Charged particles}

In proton therapy breast treatments, SGRT combined with initial and weekly in-beam X-ray imaging, has been proven to be a safe replacement of daily orthogonal Xray images for patient positioning, resulting in shorter setup time, and reduction of imaging dose for the patient $[14,58,89]$. With this approach, significant discrepancies $(>3 \mathrm{~mm}$ shifts) between surface and radiographic imaging indicate changes in breast anatomy. A further application in proton therapy is the verification of the nozzle setup [90], by air gap and source to surface distance (SSD) calculations, which provide a way to confirm the physical target depth at treatment.

\section{Quality assurance (QA) - update on current status}

SGRT systems require a demanding Quality Assurance (QA) program in order to achieve submillimeter accuracy and reliable functioning. Not only the system performance itself, but also the associated workflow needs to be ensured. AAPM TG 147 [91] set the first guidelines about this topic. However, after 8 years of technological development and a wider usage of SGRT by an increased number of facilities, there is a need for updated QA guidelines.

Applications of hypofractionated RT (e.g. SRS, SBRT) require measurements of the static accuracy with $6 \mathrm{DOF}$ [92], differentiation between single or multiple isocenter plans [93], including couch rotations, walkout effects, and the impact of miscalibration [19,94].

Additionally, the gain in the frame-rate of the newest SGRT systems attracts the use to treat patients under free-breathing, where parameters as gating response times, gating window and Linac configurations (e.g. dose rate, beam hold times) need to be included into the QA program $[86,95,96]$. Even more complex, is the application of this technology for particle therapy, specifically in pencil beam delivery, where parameters as latency, the impact of room geometries into the system performance and potential prediction algorithms, when using beam-gating or -tracking $[97,98]$ need to be taken into account. Also in ring-like Linacs, a QA program needs to be implemented $[37,99,100]$, as well as when the surface is used as a surrogate for 4D-acquisitions [101-104]. 
Although the majority of the users use commercially available phantoms, some features are currently impossible (or challenging) to test, such as deformable registration algorithms [7, 36], latency time [105], skin colors and room lightening, full-workflow integration [106], and thermal signature.

\section{Future applications}

The number of SGRT users and applications has increased rapidly in recent years and is expected to further increase in the coming years. Considering the discomfort and emotional burden of tattoos, which could be a constant reminder of a patient's cancer treatment, a complete replacement of patient's tattooing with a markerless SGRT-based workflow could be a real prospect in the near future.

Deformable surface registration, in combination with extensive studies on the correlation of patient surface motion to internal organ and tumor motion, can constitute a more accurate method for recording and monitoring patient motion and anatomy changes. Motion monitoring for DIBH, gated or even treatments with tumor tracking, can benefit from the improved data that SGRT offers in comparison to 1D surrogates. Surface data acquired during CT simulation can help to stratify patients to motion mitigation techniques, for example by quantifying the patient's ability to follow the instructions required for a certain breath hold technique. Also, with respect to interfractional anatomical changes, such as weight loss or lymphedema in breast treatments, SGRT offers a potential. With the upcoming possibility of thermal tracking (ExacTrac Dynamic, Brainlab AG, Germany), even the judgement of physiologic processes, such as inflammation or early skin toxicity might be accessible.

Along with the retrospective analysis of patient motion, resulting from intra-fractional monitoring data, the SGRT systems provide the information of the setup variability, which combined with anatomical imaging can determine the necessary information to establish site- or even patient-specific treatment margins. It is therefore expected that SGRT will increasingly contribute to the development of personalized patient care and adaptive radiotherapy treatments, with the potential to decrease the volume of irradiated healthy tissues and the total number of verification imaging (X-ray or $\mathrm{CBCT}$ ) required to control inter-fractional variations. SGRT can either replace the $\mathrm{kV}$ imaging itself or trigger $\mathrm{kV}$ images when changes over a certain threshold are detected.

Recently, a combination of SGRT with X-ray monitoring in a dedicated system has been introduced (ExacTrac Dynamic, Brainlab AG, Germany). The combined workflow could enables SGRT-guided patient positioning and intrafractional motion monitoring with the possibility of positioning the patient also according to the internal anatomy. An automatic triggering of X-ray images allows to verify the internal position of the target, when the patient surface exceeds a certain tolerance. As an addition to the surface information, a thermal signature of the surface is used to increase registration accuracy, as shown exemplarily in Fig. 5.

The determinant role of SGRT in terms of adaptive planning can be of greater importance for particle therapy, where undetected patient anatomy and posture changes, such as inflammation, weight increase or loss, may have more substantial dosimetric effects due to the strong influence of changes in particle range $[107,108]$. However, SGRT application in particle therapy can be expected to further extend also in machine and patientspecific QA. Due to the extensive weight of the gantry configuration, the mechanical and radiation isocentricity in most proton therapy systems strongly depends on complicated algorithms to adjust the couch position as a compensation for gantry sagging, which varies according per treatment angle. A well-calibrated surface imaging system with a system-specific QA routine could be implemented as a secondary verification procedure for couch-positioning verification, but also as an independent check of gantry and couch position (and motion in case of VMAT) for photon beam therapy.

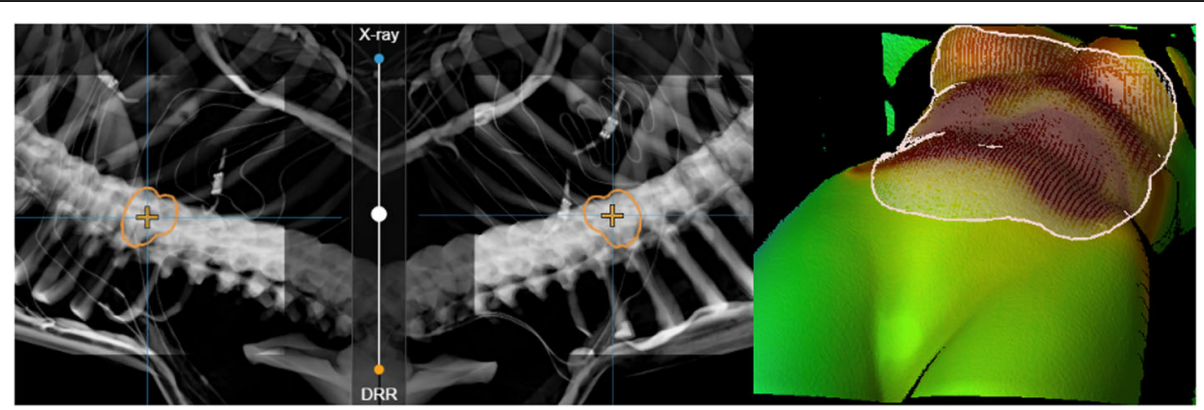

Fig. 5 A combination of X-ray monitoring (left) and SGRT guidance (right). A phantom with a distinct heat signature has been used for demonstration purposes. SGRT and X-ray tracking are combined in a single workflow. Image courtesy of LMU University Hospital Munich, Germany 
Furthermore, SGRT systems could be integrated into the entire clinical radiation therapy workflow, considering the recent advances in biometrics and face recognition algorithms. SGRT is able to provide patient registration upon arrival via facial recognition, daily verification of treatment accessory items at the linac and verify their correct position on the couch, moreover it can facilitate patient-specific and machine QA.

For each of the enumerated applications, a dedicated QA program, including the SGRT-system and the peripherals (i.e. TPS, Linac, imaging systems) must be implemented. Development of suitable phantoms, integration in an analysis framework and parameterization of the system quality are some of the fields to be explored.

\section{Conclusion}

The use of SGRT has shown to provide increased patient safety throughout the course of the radiotherapy treatment and increased accuracy for the treatment of specific anatomic sites. SGRT can be considered an additional safety tool, for example for intra-fractional motion management, but certain techniques such as DIBH or open masks treatments have made extraordinary progress through the use of SGRT.

\section{Abbreviations}

4DCT: Four-dimensional computed tomography; APBI: Accelerated partial breast irradiation; BM: Brain metastases; DIBH: Deep-Inspiration-Breath-hold; CBCT: Cone-beam computed tomography; CT: Computed tomography?; FFF: Flattening filter free; NAL : No Action Level; QA: Quality Assurance; ROI: Region-of interest; PTV: Planning target volume; SBRT: Stereotactic body radiation therapy; SGRT: Surface Guided Radiation Therapy; SRS: Stereotactic radiosurgery; SSD: Source to surface distance; TRE: Target registration error; VDIBH: Voluntary-DIBH; WBRT: Whole-brain radiation therapy
\end{abstract}

\section{Acknowledgements}

Not applicable.

\section{Authors' contributions}

PF \& VB designed the manuscript. PF wrote parts of introduction, the section "Breath-hold", and the conclusion. MK \& TOS wrote sections "Breast positioning", "Positioning of Internal targets (Abdomen/Pelvis/Head \& Neck)", and parts of the introduction. MÖ \& AS wrote the section "Intracranial tumors: WBRT \& SRS". GD wrote sections "Future applications" \& "Special techniques". CB reviewed sections "Future applications" \& "Special techniques". SC revised and reviewed the manuscript and contributed to the section "Breath-hold". VB wrote the abstract, parts of the introduction and the section "Quality Assurance (QA) - Update on Current Status". All authors read and approved the final manuscript.

\section{Funding}

Not applicable.

\section{Availability of data and materials}

Not applicable.

Ethics approval and consent to participate

Not applicable.

Consent for publication

Not applicable.

\section{Competing interests}

PF, SC has received research grants from C-RAD AB and Brainlab AG. PF has received speaker honoraria from $C-R A D A B$ and Brainlab AG. SC, AS, and MÖ have received speaker honoraria from C-RAD AB. VB represents her Hospital in a cooperation agreement and in a reference site agreement with Vision RT.

\section{Author details}

${ }^{1}$ Department of Radiation Oncology, University Hospital, LMU Munich, Munich, Germany. 'Department of Hematology, Oncology and Radiation Physics, Skåne University Hospital, Lund, Sweden. ${ }^{3}$ Medical Radiation Physics, Department of Clinical Sciences, Lund University, Lund, Sweden. ${ }^{4}$ Maastricht Radiation Oncology (MAASTRO), Maastricht, the Netherlands. ${ }^{5}$ Department of Radiation Oncology, Universitätsklinikum Erlangen,

Friedrich-Alexander-Universität Erlangen-Nürnberg (FAU), Erlangen, Germany.

${ }^{6}$ Department of Radiation Oncology, Massachusetts General Hospital and Harvard Medical School, Boston, USA. ${ }^{7}$ Department of Radiation Oncology, Heidelberg University Hospital, Heidelberg, Germany. ${ }^{8}$ Heidelberg Institute of Radiation Oncology (HIRO), Heidelberg, Germany. ${ }^{9}$ National Center for Tumor diseases (NCT), Heidelberg, Germany.

Received: 28 May 2020 Accepted: 21 July 2020

Published online: 31 July 2020

\section{References}

1. Brahme A, Nyman P, Skatt BB. 4D laser camera for accurate patient positioning, collision avoidance, image fusion and adaptive approaches during diagnostic and therapeutic procedures. Med Phys. 2008;35(5):1670.

2. Placht S, Stancanello J, Schaller C, Balda M, Angelopoulou E. Fast time-offlight camera based surface registration for radiotherapy patient positioning. Med Phys. 2012;39(1):4-17.

3. Pycinski B, Czajkowska J, Badura P, Juszczyk J, Pietka E. Time-of-flight camera, optical tracker and computed tomography in pairwise data registration. PLoS One. 2016;11(7):1-20.

4. Bert C, Metheany KG, Doppke K, Chen GTY. A phantom evaluation of a stereo-vision surface imaging system for radiotherapy patient setup. Med Phys. 2005;32(9):2753-62.

5. Lindl BL, Müller RG, Lang S, Herraiz Lablanca MD, Klöck S. TOPOS: a new topometric patient positioning and tracking system for radiation therapy based on structured white light. Med Phys. 2013;40(4):1-10.

6. Nutti B, Kronander A, Mattias N, Maad K, Cristina S, Li H. Depth Sensor-Based Realtime Tumor Tracking for Accurate Radiation Therapy. Proc Eurographics Short Pap. 2014;2014:10-3.

7. Pallotta S, Marrazzo L, Ceroti M, Silli P, Bucciolini M. A phantom evaluation of sentinel, a commercial laser/camera surface imaging system for patient setup verification in radiotherapy. Med Phys. 2012;39(2):706-12.

8. Hoisak JDP, Pawlicki T. The role of optical surface imaging Systems in Radiation Therapy. Semin Radiat Oncol. 2018 Jul;28(3):185-93.

9. Manger RP, Paxton AB, Pawlicki T, Kim GY. Failure mode and effects analysis and fault tree analysis of surface image guided cranial radiosurgery. Med Phys. 2015 May 1;42(5):2449-61.

10. Rusu I, Thomas TO, Roeske JC, Mescioglu I, Melian E, Surucu M. Failure mode and effects analysis of linac-based liver stereotactic body radiotherapy. Med Phys. 2020:mp.13965.

11. Lau SKM, Patel K, Kim T, Knipprath E, Kim GY, Cerviño LI, et al. Clinical efficacy and safety of surface imaging guided radiosurgery (SIG-RS) in the treatment of benign skull base tumors. J Neuro-Oncol. 2017 Apr 1;132(2): 307-12.

12. Zhao B, Maquilan G, Jiang S, Schwartz DL. Minimal mask immobilization with optical surface guidance for head and neck radiotherapy. J Appl Clin Med Phys. 2018;19(1):17-24.

13. Stanley DN, Mcconnell KA, Kirby N, Gutiérrez AN, Papanikolaou N, Rasmussen K. Comparison of initial patient setup accuracy between surface imaging and three point localization: a retrospective analysis. J Appl Clin Med Phys. 2017 Nov 1;18(6):58-61.

14. Batin E, Depauw N, MacDonald S, Lu H-MM. Can surface imaging improve the patient setup for proton postmastectomy chest wall irradiation? Pract Radiat Oncol. 2016 Nov 1;6(6):e235-41.

15. Piroth MD, Baumann R, Budach W, Dunst J, Feyer P, Fietkau R, et al. Heart toxicity from breast cancer radiotherapy: current findings, assessment, and 
prevention, vol. 195. Urban und Vogel GmbH: Strahlentherapie und Onkologie; 2019.

16. Kügele M, Mannerberg A, Nørring Bekke S, Alkner S, Berg L, Mahmood F, et al. Surface guided radiotherapy (SGRT) improves breast cancer patient setup accuracy. J Appl Clin Med Phys. 2019;20(9):61-8.

17. Kost S, Shah CS, Xia P, Guo B. Setup time and positioning accuracy in breast radiation therapy using surface guided radiation therapy. Int J Radiat Oncol. 2018 Nov;102(3):e481-2.

18. Murphy MJ, Balter J, Balter S, Jiang SB. The management of imaging dose during image-guided radiotherapy : Report of the AAPM Task Group 75. 2007;34:4041.

19. Swinnen ACC, Öllers MC, Ong CLVF. The potential of an optical surface tracking system in non-coplanar SRS treatments of single isocenter multiple brain metastases. J Appl Clin Med Phys. 2020; Accepted f.

20. Hoisak JDP, Paxton AB, Waghorn BJ, Pawlicki T. Surface Guided Radiation Therapy. Definitions: CRC Press; 2020.

21. Zhao H, Williams N, Poppe M, Sarkar V, Wang B, Rassiah-Szegedi $P$, et al. Comparison of surface guidance and target matching for image-guided accelerated partial breast irradiation (APBI). Med Phys. 2019 Nov 4;46(11): 4717-24.

22. Carl G, Reitz D, Schönecker S, Pazos M, Freislederer P, Reiner M, et al. Optical surface scanning for patient positioning in radiation therapy: a prospective analysis of 1902 fractions. Technol Cancer Res Treat. 2018:17: 1533033818806002.

23. Bert C, Metheany KG, Doppke KP, Taghian AG, Powell SN, Chen GTY. Clinical experience with a 3D surface patient setup system for alignment of partialbreast irradiation patients. Int J Radiat Oncol Biol Phys. 2006 Mar 15;64(4): 1265-74.

24. Gierga DP, Riboldi M, Turcotte JC, Sharp GC, Jiang SB, Taghian AG, et al. Comparison of target registration errors for multiple image-guided techniques in accelerated partial breast irradiation. Int J Radiat Oncol. 2008 Mar;70(4):1239-46.

25. Chang AJ, Zhao H, Wahab SH, Moore K, Taylor M, Zoberi I, et al. Video surface image guidance for external beam partial breast irradiation. Pract Radiat Oncol. 2012 Apr;2(2):97-105.

26. Jimenez RB, Batin E, Giantsoudi D, Hazeltine W, Bertolino K, Ho AY, et al. Tattoo free setup for partial breast irradiation: a feasibility study. J Appl Clin Med Phys. 2019 Apr 4;20(4):45-50

27. Shah AP, Dvorak T, Curry MS, Buchholz DJ, Meeks SL. Clinical evaluation of interfractional variations for whole breast radiotherapy using 3-dimensional surface imaging. Pract Radiat Oncol. 2013 Jan;3(1):16-25.

28. Padilla L, Kang H, Washington M, Hasan Y, Chmura SJ, Al-Hallaq H. Assessment of interfractional variation of the breast surface following conventional patient positioning for whole-breast radiotherapy. J Appl Clin Med Phys. 2014;15(5):177-89.

29. Hattel SH, Andersen PA, Wahlstedt IH, Damkjær S, Saini A, Thomsen JB. Evaluation of setup and intrafraction motion for surface guided whole-breast cancer radiotherapy. J Appl Clin Med Phys. 2019 Jun 1; 20(6):39-44

30. Laaksomaa M, Sarudis S, Rossi M, Lehtonen T, Pehkonen J, Remes J, et al. AlignRT ${ }^{\circledR}$ and catalyst $^{\mathrm{TM}}$ in whole-breast radiotherapy with DIBH: is IGRT still needed? J Appl Clin Med Phys. 2019;20(3):97-104.

31. Haraldsson A, Ceberg S, Crister C, Engelholm S, Bäck SÅJ, Engström PE. PO0978 accurate positioning with decreased treatment time using surface guided tomotherapy. Radiother Oncol. 2019 Apr 1;133:S534-5.

32. Padilla L, Havnen-Smith A, Cerviño L, Al-Hallaq HA. A survey of surface imaging use in radiation oncology in the United States. J Appl Clin Med Phys. 2019 Dec 19;20(12):70-7.

33. Reitz D, Carl G, Schönecker S, Pazos M, Freislederer P, Niyazi M, et al. Realtime intra-fraction motion management in breast cancer radiotherapy: analysis of 2028 treatment sessions. Radiat Oncol. 2018;13(1):1-9.

34. Pazos M, Walter F, Reitz D, Schönecker $S$, Konnerth D, Schäfer A, et al. Impact of surface-guided positioning on the use of portal imaging and initial set-up duration in breast cancer patients. Strahlentherapie und Onkol. 2019;195(11):964-71

35. Baroni G, Ferrigno G, Orecchia R, Pedotti A. Real-time three-dimensional motion analysis for patient positioning verification. Radiother Oncol. 2000; 54(1):21-7.

36. Meyer J, Smith W, Geneser S, Koger B, Kalet AM, Young LA, et al. Characterizing a deformable registration algorithm for surface-guided breast radiotherapy. Med Phys. 2019;47:352 mp.13921.
37. Crop F, Pasquier D, Baczkiewic A, Doré J, Bequet L, Steux E, et al. Surface imaging, laser positioning or volumetric imaging for breast cancer with nodal involvement treated by helical TomoTherapy. J Appl Clin Med Phys. 2016 Sep;17(5):200-11.

38. Walter F, Freislederer P, Belka C, Heinz C, Söhn M, Roeder F. Evaluation of daily patient positioning for radiotherapy with a commercial 3D surfaceimaging system (Catalyst ${ }^{\mathrm{TM}}$ ). Radiat Oncol. 2016;11:1.

39. Wikström K, Nilsson K, Isacsson U, Ahnesjö A. A comparison of patient position displacements from body surface laser scanning and cone beam CT bone registrations for radiotherapy of pelvic targets. Acta Oncol (Madr). 2014 Feb;53(2):268-77.

40. Pinkham MB, Sanghera P, Wall GK, Dawson BD, Whitfield GA. Neurocognitive effects following cranial irradiation for brain metastases. Clin Oncol. 2015 Nov 1;27(11):630-9.

41. Zindler JD, Bruynzeel AME, Eekers DBP, Hurkmans CW, Swinnen A, Lambin P. Whole brain radiotherapy versus stereotactic radiosurgery for 4-10 brain metastases: a phase III randomised multicentre trial. BMC Cancer. 2017 Jul;25:17(1)

42. Kraft J, Zindler J, Minniti G, Guckenberger M, Andratschke N. Stereotactic radiosurgery for multiple brain metastases. In: Current Treatment Options in Neurology, vol. 21: Current Science Inc.; 2019.

43. Ruggieri R, Naccarato S, Mazzola R, Ricchetti F, Corradini S, Fiorentino A, et al. Linac-based VMAT radiosurgery for multiple brain lesions: comparison between a conventional multi-isocenter approach and a new dedicated mono-isocenter technique. Radiat Oncol. 2018 Mar 5;13(1):38.

44. Hartgerink D, Swinnen A, Roberge D, Nichol A, Zygmanski P, Yin F-F, et al. LINAC based stereotactic radiosurgery for multiple brain metastases: guidance for clinical implementation. Acta Oncol. 2019 Sep;58(9):1275-82.

45. Wen N, Snyder KC, Scheib SG, Schmelzer P, Qin Y, Li H, et al. Technical note: evaluation of the systematic accuracy of a frameless, multiple image modality guided, linear accelerator based stereotactic radiosurgery system. Med Phys. 2016 May;43(5):2527

46. Kirkpatrick JP, Wang Z, Sampson JH, McSherry F, Herndon JE, Allen KJ, et al. Defining the optimal planning target volume in image-guided stereotactic radiosurgery of brain metastases: results of a randomized trial. Int J Radiat Oncol Biol Phys. 2015 Jan 1;91(1):100-8.

47. Alongi F, Fiorentino A, Gregucci F, Corradini S, Giaj-Levra N, Romano L, et al. First experience and clinical results using a new non-coplanar monoisocenter technique (HyperAr ${ }^{\mathrm{TM}}$ ) for Linac-based VMAT radiosurgery in brain metastases. J Cancer Res Clin Oncol. 2019 Jan 22;145(1):193-200.

48. Hofmaier J, Bodensohn R, Garny S, Hadi I, Fleischmann DF, Eder M, et al. Single isocenter stereotactic radiosurgery for patients with multiple brain metastases: Dosimetric comparison of VMAT and a dedicated DCAT planning tool. Radiat Oncol. 2019;14(1):4-11.

49. Ruggieri R, Naccarato S, Mazzola R, Ricchetti F, Corradini S, Fiorentino A, et al. Linac-based radiosurgery for multiple brain metastases: comparison between two mono-isocenter techniques with multiple non-coplanar arcs. Radiother Oncol. 2019 Mar 1:132:70-8.

50. Oh SA, Yea JW, Kang MK, Park JW, Kim SK. Analysis of the setup uncertainty and margin of the daily ExacTrac 6D image guide system for patients with brain tumors. PLoS One. 2016;11(3):e0151709.

51. Lewis BC, Snyder WJ, Kim S, Kim T. Monitoring frequency of intra-fraction patient motion using the ExacTrac system for LINAC-based SRS treatments. J Appl Clin Med Phys. 2018 May 1:19(3):58-63.

52. Tarnavski N, Engelholm SA, Af Rosenschold PM. Fast intra-fractional imageguidance with 6D positioning correction reduces delivery uncertainty for stereotactic radiosurgery and radiotherapy. J radiosurgery SBRT. 2016;4(1):15-20.

53. Dekker J, Rozema T, Böing-Messing F, Garcia M, Washington D, de Kruijf W. Whole-brain radiation therapy without a thermoplastic mask. Phys Imaging Radiat Oncol. 2019 Jul 1;11:27-9.

54. Du W, Johnson JL, Jiang W, Kudchadker RJ. On the selection of gantry and collimator angles for isocenter localization using Winston-Lutz tests. J Appl Clin Med Phys. 2016;17(1):167-78.

55. Covington EL, Fiveash JB, Wu X, Brezovich I, Willey CD, Riley K, et al. Optical surface guidance for submillimeter monitoring of patient position during frameless stereotactic radiotherapy. J Appl Clin Med Phys. 2019 Jun;20(6):91-8.

56. Boda-Heggemann J, Knopf AC, Simeonova-Chergou A, Wertz H, Stieler F, Jahnke A, et al. Deep inspiration breath hold - based radiation therapy: a clinical review. Int J Radiat Oncol Biol Phys. 2016;94(3):478-92.

57. Bergom C, Currey A, Desai N, Tai A, Strauss JB. Deep inspiration breath hold: Techniques and advantages for cardiac sparing during breast cancer irradiation. Front Oncol. 2018;8(APR):1-10. 
58. Batin E, Depauw N, Jimenez RB, MacDonald S, Lu H-M. Reducing X-ray imaging for proton postmastectomy chest wall patients. Pract Radiat Oncol. 2018 Sep;8(5):e266-74

59. Darby SC, Ewertz M, McGale P, Bennet AM, Blom-Goldman U, Brnønum D, et al. Risk of ischemic heart disease in women after radiotherapy for breast cancer. N Engl J Med. 2013;368(11):987-98.

60. Simonetto C, Eidemüller M, Gaasch A, Pazos M, Schönecker S, Reitz D, et al. Does deep inspiration breath-hold prolong life? Individual risk estimates of ischaemic heart disease after breast cancer radiotherapy. Radiother Oncol. 2019;131:202-7.

61. Corradini S, Ballhausen H, Weingandt H, Freislederer P, Schönecker S, Niyazi $M$, et al. Left-sided breast cancer and risks of secondary lung cancer and ischemic heart disease: effects of modern radiotherapy techniques. Strahlentherapie und Onkol. 2018;194(3):196-205.

62. Pazos M, Fiorentino A, Gaasch A, Schönecker S, Reitz D, Heinz C, et al. Dose variability in different lymph node levels during locoregional breast cancer irradiation: the impact of deep-inspiration breath hold. Strahlentherapie und Onkol. 2019;195(1):13-20.

63. Gaasch A, Schönecker S, Simonetto C, Eidemüller M, Pazos M, Reitz D, et al. Heart sparing radiotherapy in breast cancer: the importance of baseline cardiac risks. Radiat Oncol. 2020 Dec 24;15(1):117.

64. Lin LL, Vennarini S, Dimofte A, Ravanelli D, Shillington K, Batra S, et al. Proton beam versus photon beam dose to the heart and left anterior descending artery for left-sided breast cancer. Acta Oncol (Madr). 2015;54(7): 1032-9.

65. Edvardsson A, Kügele M, Alkner S, Enmark M, Nilsson J, Kristensen I, et al. Comparative treatment planning study for mediastinal Hodgkin's lymphoma: impact on normal tissue dose using deep inspiration breath hold proton and photon therapy. Acta Oncol (Madr). 2019;58(1):95-104.

66. Schönecker S, Walter F, Freislederer P, Marisch C, Scheithauer H, Harbeck N, et al. Treatment planning and evaluation of gated radiotherapy in left-sided breast cancer patients using the CatalystTM/SentinelTM system for deep inspiration breath-hold (DIBH). Radiat Oncol. 2016;11(1):143.

67. Pandeli C, Smyth LML, David S, See AW. Dose reduction to organs at risk with deep-inspiration breath-hold during right breast radiotherapy: a treatment planning study. Radiat Oncol. 2019;14(1):1-10.

68. Xiao A, Crosby J, Malin M, Kang H, Washington M, Hasan Y, et al. Singleinstitution report of setup margins of voluntary deep-inspiration breathhold (DIBH) whole breast radiotherapy implemented with real-time surface imaging. J Appl Clin Med Phys. 2018;19(4):205-13.

69. Reitz D, Walter F, Schönecker S, Freislederer P, Pazos M, Niyazi M, et al. Stability and reproducibility of 6013 deep inspiration breath-holds in leftsided breast cancer. Radiat Oncol. 2020 Dec 24;15(1):121.

70. Kügele M, Edvardsson A, Berg L, Alkner S, Andersson Ljus C, Ceberg S. Dosimetric effects of intrafractional isocenter variation during deep inspiration breath-hold for breast cancer patients using surface-guided radiotherapy. J Appl Clin Med Phys. 2018;19(1):25-38.

71. Hamming VC, Visser C, Batin E, McDermott LN, Busz DM, Both S, et al. Evaluation of a 3D surface imaging system for deep inspiration breath-hold patient positioning and intra-fraction monitoring. Radiat Oncol. 2019;14(1):4-11.

72. MacDonald SM, Gierga DP, Napolitano B, Taghian AG. APBI 3D Conformal External Beam: The MGH Technique. In: Accelerated Partial Breast Irradiation. Berlin, Heidelberg: Springer Berlin Heidelberg; 2009. p. 315-26.

73. Leong B, Padilla L. Impact of use of optical surface imaging on initial patient setup for stereotactic body radiotherapy treatments. J Appl Clin Med Phys. 2019 Dec 13;20(12):149-58.

74. Alderliesten T, Sonke J-J, Betgen A, van Vliet-Vroegindeweij C, Remeijer P. $3 \mathrm{D}$ surface imaging for monitoring intrafraction motion in frameless stereotactic body radiotherapy of lung cancer. Radiother Oncol. 2012 Nov; 105(2):155-60.

75. Spadea MF, Baroni G, Gierga DP, Turcotte JC, Chen GTY, Sharp GC Evaluation and commissioning of a surface based system for respiratory sensing in 4D CT. J Appl Clin Med Phys. 2011 Dec;12(1):162-9.

76. Kauweloa Kl, Ruan D, Park JC, Sandhu A, Kim GY, Pawlicki T, et al. GateCT'm surface tracking system for respiratory signal reconstruction in 4DCT imaging. Med Phys. 2011 Dec 30;39(1):492-502.

77. Jin H, Su Z. SU-E-T-225: A Comprehensive Evaluation of Real-Time Motion Tracking of a Surface Imaging System for Lung Treatment. Med Phys. 2012; 39(6Part13):3755

78. Schaerer J, Fassi A, Riboldi M, Cerveri P, Baroni G, Sarrut D. Multidimensional respiratory motion tracking from markerless optical surface imaging based on deformable mesh registration. Phys Med Biol. 2012 Jan 21;57(2):357-73.

79. Glide-Hurst CK, lonascu D, Berbeco R, Yan D. Coupling surface cameras with on-board fluoroscopy: A feasibility study. Med Phys. 2011;38(6Part1):2937-47.

80. Fassi A, Schaerer JJ, Fernandes M, Riboldi MM, Sarrut D, Baroni G. Tumor tracking method based on a deformable 4D CT breathing motion model driven by an external surface surrogate. Int J Radiat Oncol. 2014 Jan;88(1): $182-8$.

81. Hughes S, McClelland J, Tarte S, Lawrence D, Ahmad S, Hawkes D, et al. Assessment of two novel ventilatory surrogates for use in the delivery of gated/tracked radiotherapy for non-small cell lung cancer. Radiother Oncol. 2009 Jun;91(3):336-41.

82. Li G, Huang H, Wei J, Li DG, Chen Q, Gaebler CP, et al. Novel spirometry based on optical surface imaging. Med Phys. 2015 Mar 18;42(4):1690-7.

83. Li G, Wei J, Huang H, Chen Q, Gaebler CP, Lin T, et al. Characterization of optical-surface-imaging-based spirometry for respiratory surrogating in radiotherapy. Med Phys. 2016 Feb 23:43(3):1348-60.

84. Freislederer P, Reiner M, Hoischen W, Quanz A, Heinz C, Walter F, et al. Characteristics of gated treatment using an optical surface imaging and gating system on an Elekta linac. Radiat Oncol. 2015;10(1):1-6.

85. Wiersma RD, McCabe BP, Belcher AH, Jensen PJ, Smith B, Aydogan B. Technical Note: High temporal resolution characterization of gating response time. Med Phys. 2016:43(6Part1):2802-6.

86. Barfield G, Burton EW, Stoddart J, Metwaly M, Cawley MG. Quality assurance of gating response times for surface guided motion management treatment delivery using an electronic portal imaging detector. Phys Med Biol. 2019 Jun 21;64(12):125023.

87. Rwigema J-CM, Lamiman K, Reznik RS, Lee NJH, Olch A, Wong KK. Palliative radiation therapy for superior vena cava syndrome in metastatic Wilms tumor using 10XFFF and 3D surface imaging to avoid anesthesia in a pediatric patient-a teaching case. Adv Radiat Oncol. 2017 Jan;2(1):101-4.

88. Sueyoshi M, Olch AJ, Liu KX, Chlebik A, Clark D, Wong KK. Eliminating daily shifts, tattoos, and skin Marks: streamlining Isocenter localization with treatment plan embedded couch values for external beam radiation therapy. Pract Radiat Oncol. 2019;9(1):e110-7.

89. Depauw N, Batin E, Daartz J, Rosenfeld A, Adams J, Kooy H, et al. A novel approach to Postmastectomy radiation therapy using scanned proton beams. Int J Radiat Oncol. 2015 Feb;91(2):427-34.

90. Wang X, Ma C, Davis R, Parikh RR, Jabbour SK, Haffty BG, et al. A novel approach to Verify air gap and SSD for proton radiotherapy using surface imaging. Radiat Oncol. 2019 Dec 11;14(1):224.

91. Willoughby T, Lehmann J, Bencomo JA, Jani SK, Santanam L, Sethi A, et al Quality assurance for nonradiographic radiotherapy localization and positioning systems: report of task group 147. Med Phys. 2012 Apr;39(4): 1728-47.

92. Belcher AH, Liu X, Grelewicz Z, Wiersma RD. Spatial and rotational quality assurance of 6DOF patient tracking systems. Med Phys. 2016 Jun 1;43(6): 2785-93.

93. Oliver JA, Kelly P, Meeks SL, Willoughby TR, Shah AP. Orthogonal image pairs coupled with OSMS for noncoplanar beam angle, intracranial, singleisocenter, SRS treatments with multiple targets on the Varian Edge radiosurgery system. Adv Radiat Oncol. 2(3):494-502.

94. Paxton AB, Manger RP, Pawlicki T, Kim GY. Evaluation of a surface imaging system's isocenter calibration methods. J Appl Clin Med Phys. 2017 Mar 1; 18(2):85-91.

95. Freislederer P. Gating characteristics of the catalyst TM surface imaging system in combination with the Elekta response TM Interface. STRAHLEN THERAPIE UND Onkol. 2014 Jul;190(1):1-13.

96. Cui G, Housley DJ, Chen F, Mehta VK, Shepard DM. Delivery efficiency of an Elekta linac under gated operation. J Appl Clin Med Phys. 2014;15(5):2-11.

97. Fattori G, Seregni M, Pella A, Riboldi M, Capasso L, Donetti M, et al. Realtime optical tracking for motion compensated irradiation with scanned particle beams at CNAO. Nucl Instruments Methods Phys Res Sect A Accel Spectrometers, Detect Assoc Equip. 2016:827:39-45.

98. Fattori G, Safai S, Carmona PF, Peroni M, Perrin R, Weber DC, et al. Monitoring of breathing motion in image-guided PBS proton therapy: comparative analysis of optical and electromagnetic technologies. Radiat Oncol. 2017 Mar 31;12(1):63.

99. Wiencierz M, Kruppa K, Lüdemann L. Clinical validation of two surface imaging Systems for Patient Positioning in percutaneous radiotherapy; 2016 Feb 11 . 
100. Flores-Martinez E, Cerviño LI, Pawlicki T, Kim G-Y. Assessment of the use of different imaging and delivery techniques for cranial treatments on the Halcyon linac. J Appl Clin Med Phys. 2020 Jan;21(1):53-61.

101. Jönsson M, Ceberg S, Nordström F, Thornberg C, Bäck SAJ. Technical evaluation of a laser-based optical surface scanning system for prospective and retrospective breathing adapted computed tomography. Acta Oncol (Madr). 2015;54(2):261-5.

102. Delombaerde L, Petillion S, Michiels S, Weltens C, Depuydt T. Development and accuracy evaluation of a single-camera intra-bore surface scanning system for radiotherapy in an O-ring linac. Phys Imaging Radiat Oncol. 2019 Jul 1;11:21-6.

103. Heß M, Büther F, Gigengack F, Dawood M, Schäfers KP. A dual-Kinect approach to determine torso surface motion for respiratory motion correction in PET. Med Phys. 2015 May:42(5):2276-86.

104. Fassi A, Schaerer J, Riboldi M, Sarrut D, Baroni G. An image-based method to synchronize cone-beam CT and optical surface tracking. J Appl Clin Med Phys. 2015 Mar 8;16(2):5152.

105. Lempart M, Kügele M, Ambolt L, Blad B, Nordström F. Latency characterization of gated radiotherapy treatment beams using a PIN diode circuit. IRBM. 2016 Jun 1;37(3):144-51.

106. Yu AS, Fowler TL, Dubrowski P. A novel-integrated quality assurance phantom for radiographic and nonradiographic radiotherapy localization and positioning systems. Med Phys. 2018 Jul;45(7):2857-63.

107. Landry G, Hua C. Current state and future applications of radiological image guidance for particle therapy. Med Phys. 2018 Nov 13;45(11):e1086-95.

108. Seco J, Spadea MF. Imaging in particle therapy: state of the art and future perspective. Acta Oncol (Madr). 2015 Oct 21;54(9):1254-8.

\section{Publisher's Note}

Springer Nature remains neutral with regard to jurisdictional claims in published maps and institutional affiliations.

Ready to submit your research? Choose BMC and benefit from:

- fast, convenient online submission

- thorough peer review by experienced researchers in your field

- rapid publication on acceptance

- support for research data, including large and complex data types

- gold Open Access which fosters wider collaboration and increased citations

- maximum visibility for your research: over $100 \mathrm{M}$ website views per year

At $\mathrm{BMC}$, research is always in progress.

Learn more biomedcentral.com/submissions 